\title{
Power corrections to pion transition form factor in perturbative QCD approach
}

\author{
Yue-Long Shen, ${ }^{1, *}$ Zhi-Tian Zou, ${ }^{2, \dagger}$ and Ying $\mathrm{Li}^{2,+}$ \\ ${ }^{1}$ College of Information Science and Engineering, Ocean University of China, Qingdao, \\ Shandong 266100, People's Republic of China \\ ${ }^{2}$ Department of Physics, Yantai University, Yantai 264005, China
}

(Received 18 January 2019; published 30 July 2019)

\begin{abstract}
In this paper we calculate the power corrections to the pion transition form factor within the framework of the perturbative QCD approach on the basis of $k_{T}$ factorization. The power suppressed contributions from higher-twist pion wave functions and the hadronic structure of photon are investigated. We find that there exists strong cancellation between the two kinds of contributions; thus, the total power corrections considered currently are very small, and the prediction of the leading power contribution with jointresummation improved perturbative QCD approach is almost unchanged. This result confirms that the pion transition form factor is a good platform to constrain the nonperturbative parameters in pion wave functions. Moreover, our result can accommodate the anomalous data from $B A B A R$, or agrees with results from Belle according to the choice of Gegenbauer moment in the pion wave function, and the more precise experimental data from Belle-II is expected.
\end{abstract}

DOI: 10.1103/PhysRevD.100.016022

\section{INTRODUCTION}

The pion-photon transition process $\gamma^{*} \gamma \rightarrow \pi^{0}$ provides a golden place to test the strong interaction dynamics of hadronic reactions in the framework of QCD [1-7]. The asymptotic and soft behaviors of the pion transition form factor (TFF) $F_{\gamma^{*} \gamma \rightarrow \pi^{0}}\left(Q^{2}\right)$ have been already given as

$$
\begin{gathered}
\lim _{Q^{2} \rightarrow \infty} Q^{2} F_{\gamma^{*} \gamma \rightarrow \pi^{0}}\left(Q^{2}\right)=\sqrt{2} f_{\pi}=0.185, \\
\lim _{Q^{2} \rightarrow 0} F_{\gamma^{*} \gamma \rightarrow \pi^{0}}\left(Q^{2}\right)=\frac{\sqrt{2}}{4 \pi^{2} f_{\pi}},
\end{gathered}
$$

where $Q^{2}$ stands for the momentum transfer squared carried by the virtual photon, and the pion decay constant is $f_{\pi}=0.131 \mathrm{GeV}$. The former had been predicted within perturbative QCD in the collinear factorization theorem, and the latter one could be determined from the axial anomaly in the chiral limit. In 2009, the experimental result of BABAR [8] on $F_{\gamma^{*} \gamma \rightarrow \pi^{0}}\left(Q^{2}\right)$ exhibits an intriguing

\footnotetext{
*shenylmeteor@ouc.edu.cn

†ouzt@ytu.edu.cn

*liying@ytu.edu.cn
}

Published by the American Physical Society under the terms of the Creative Commons Attribution 4.0 International license. Further distribution of this work must maintain attribution to the author(s) and the published article's title, journal citation, and DOI. Funded by SCOAP. dependence on $Q^{2}$ : for $Q^{2}>10 \mathrm{GeV}^{2}, F_{\gamma^{*} \gamma \rightarrow \pi^{0}}\left(Q^{2}\right)$ lies above Eq. (1) and continues to grow up to $Q^{2} \approx 40 \mathrm{GeV}^{2}$. In contrast to $B A B A R$, Belle also presented their measurements in the region $4 \mathrm{GeV}^{2} \leq Q^{2} \leq 40 \mathrm{GeV}^{2}$ [9], and the rapid growth in the higher $Q^{2}$ region does not appear. Since there is no final confirmation on this discrepancy, various phenomenological approaches as well as lattice QCD simulations (see for instance [10-12]) have been employed to explain the data.

Several theoretical groups have attempted to provide explanations for the anomalous $B A B A R$ data at high $Q^{2}$ presuming that they are accurate [13-16]. In [13,14] an "exotic" twist-two pion light-cone distribution amplitude (LCDA) with the nonvanishing end-point behaviors are introduced to reproduce the scaling behavior of $B A B A R$ data. This strategy was found to be equivalent to the introduction of a sizable nonperturbative soft correction from the transverse momentum dependent (TMD) pion wave function [15]. Actually, the "exotic" wave function is not necessary in the framework of $k_{T}$ factorization [16]. At leading power the pion TFF has been studied with the Perturbative QCD(PQCD) approach based on the $k_{T}$ factorization at the one-loop level [17-19]. In the PQCD approach, resummation of the large TMD logarithms and the threshold logarithms leads to the Sudakov factor and the jet function, and an appropriate parametrization of the latter one can be used to explain the BABAR data. To avoid light-cone singularity in the TMD wave function, an offlight-cone vector is included in the definition of the wave function $[20,21]$, then additional rapidity logarithms 
arise. ${ }^{1}$ Taking advantage of joint-resummation technique one can resum the large logarithms $\ln ^{2} k_{\perp}^{2} / Q^{2}, \ln ^{2} x$, and $\ln ^{2} \zeta^{2}$ simultaneously [25]. In the joint-resummation improved factorization approach the next-to-leading order (NLO) corrections enhance the large $Q^{2}$ region, and the $B A B A R$ data can be accommodated by adjusting the Gegenbauer moment of twist-2 pion LCDA.

Confronting the theoretical predictions with the precision experimental measurements of the pion TFF at accessible $Q^{2}$ necessitates a better understanding of power suppressed contributions, especially at the mid-low- $Q^{2}$ region. In $[15,26,27]$, the light-cone sum rules (LCSR) approach is employed to calculate the soft correction to the leading twist contribution, which can effectively take the subleading power "hadronic" photon correction into account. Within this method the theoretical accuracy for predicting the pion TFF is improved by including the nextto-next-to-leading order (NNLO) QCD correction to the twist-2 contribution, the contribution from twist-4 and twist-6 pion LCDAs, and the finite-width effect of the unstable vector mesons in the hadronic dispersion relation [28-32]. In a recent study [33], the pion TFF is considered through LCSR in combination with the renormalization group of QCD, which amounts to a certain version of fractional analytic perturbation theory. Another approach to accommodate the contribution from the "hadronic photon" is to introduce the LCDAs of the photon. In [34], the LCSR for the hadronic photon contribution to the pion TFF is obtained with NLO QCD corrections to the correlation function and resummation of next-to-leading logarithms. There is strong cancellation between the contribution from twist-4 pion LCDAs and that from the leading twist LCDA of the photon, which makes the overall power correction not significant. The LCDAs of the photon, including both the two-particle and three-particle Fock states, have been studied up to the twist-4 level [35]. We note that the contribution from the higher-twist LCDAs of the photon which is not considered in the present paper might give sizable contributions [36-38].

The higher power corrections to the pion TFF have not been investigated within the $k_{T}$ factorization framework till now. Power suppressed contributions in general suffer from endpoint singularity which will make collinear factorization break down. Alternative approaches such as LCSR need to be employed, but large uncertainty arises when

\footnotetext{
${ }^{1}$ Actually there is additional self-energy divergence attributed to the infinitely long dipolar Wilson lines existing [22] after the off-light-cone vector is adopted, and a more complicated definition of TMD wave function with the dipolar Wilson links and the complicated soft subtraction [23], or with nondipolar offlight-cone Wilson links [24], is required. The definition of the wave function is essential in the soft subtraction, while for phenomenological application, it is more important to extract the hard kernel; thus, the traditional definition is still adopted in the present paper.
}

quark-hadron duality assumption is employed. In the PQCD approach the transverse momentum of parton can regularize the endpoint singularity so that the $k_{T}$ factorization is expected to work at next-to-leading power (NLP). The aim of this article is to study the NLP contributions to the pion TFF within the PQCD approach. In the current work, we will consider two kinds of power suppressed contributions, which are from the higher-twist pion wave functions and hadronic structure of the photon, respectively.

The outline of this paper is as follows: In Sec. II we review the $k_{T}$ factorization and the joint-resummation of $F_{\gamma^{*} \gamma \rightarrow \pi^{0}}\left(Q^{2}\right)$ at the leading power. In Sec. III, the analytic calculation of the power suppressed contributions will be presented. The numerical results and discussions are given in Sec. IV. We will summarize this work in the last section.

\section{FACTORIZATION AND RESUMMATION AT LEADING POWER}

The pion TFF is defined via the matrix element

$\left\langle\pi(p)\left|j_{\mu}^{\mathrm{em}}\right| \gamma\left(p^{\prime}\right)\right\rangle=g_{\mathrm{em}}^{2} \epsilon_{\mu \nu \alpha \beta} q^{\alpha} p^{\beta} \epsilon^{\nu}\left(p^{\prime}\right) F_{\gamma^{*} \gamma \rightarrow \pi^{0}}\left(Q^{2}\right)$,

where $q=p-p^{\prime} . p$ and $p^{\prime}$ refer to the four-momentum of the pion and the on-shell photon respectively, and the electromagnetic current

$$
j_{\mu}^{\mathrm{em}}=\sum_{q} g_{\mathrm{em}} Q_{q} \bar{q} \gamma_{\mu} q
$$

In the PQCD approach, the pion TFF is factorized into the convolution of the TMD wave function of the pion and the hard kernel. The $k_{T}$ factorization theorem can be derived diagrammatically [39] by applying the eikonal approximation to collinear particles and the Ward identity to the diagram summation in the leading infrared regions. The TMD wave function of pion is defined by

$$
\begin{aligned}
\Phi\left(u, k_{T}, \zeta^{2}, \mu_{f}\right)= & \int \frac{d y^{+}}{2 \pi} \frac{d^{2} y_{T}}{(2 \pi)^{2}} e^{-i u p^{-} y^{+}+i \mathbf{k}_{T} \cdot \mathbf{y}_{T}} \\
& \times\left\langle 0\left|\bar{q}(y) W_{y}(v)^{\dagger} I_{v ; y, 0} W_{0}(v) h_{+} \gamma_{5} q(0)\right| \pi(p)\right\rangle,
\end{aligned}
$$

where $\mu_{f}$ is the factorization scale, the coordinate $y=\left(y^{+}, 0, \mathbf{y}_{T}\right)$, and $u p^{-}$and $\mathbf{k}_{T}$ are the longitudinal and transverse momenta carried by the antiquark $\bar{q}$, respectively. The Wilson line,

$$
W_{y}(v)=\mathcal{P} \exp \left[-i g \int_{0}^{\infty} d \lambda v \cdot A(y+\lambda v)\right],
$$

has been introduced for maintaining the gauge invariance, where $\mathcal{P}$ denotes the path-ordered exponential. To avoid light-cone singularity, the non-light-like vector $v$ is 
employed [20]. The transverse gauge link $I_{v ; y, 0}$ does not contribute in the covariant gauge [21].

In the NLO hard kernel of pion TFF and pion wave function, the non-light-like vector $v$ will give rise to a new rapidity parameter $\zeta^{2}=\frac{4\left(n_{-} \cdot v\right)^{2}}{v^{2}}$. For the rapidity parameter $\zeta^{2}=2$, the $k_{T}$ factorization formula at leading power of $1 / Q^{2}$ under the conventional resummations is given by [16]

$$
\begin{aligned}
F^{L P, L M}\left(Q^{2}\right)= & \frac{\sqrt{2} f_{\pi}}{3} \int_{0}^{1} d u \int_{0}^{\infty} b d b \bar{\Phi}(u, b, t) e^{-S(u, b, Q, t)} S_{t}(u, Q) \\
& \times K_{0}(\sqrt{u} Q b)\left[1-\frac{\alpha_{s}(t) C_{F}}{4 \pi}\left(3 \ln \frac{t^{2} b}{2 \sqrt{u} Q}+\gamma_{E}+2 \ln u+3-\frac{\pi^{2}}{3}\right)\right],
\end{aligned}
$$

where $t$ is the factorization scale. The Sudakov factor $S(u, b, Q, t)$ sums the double logarithm $\ln ^{2}\left(k_{T}^{2} / Q^{2}\right)$ and the single logarithm $\ln \left(t^{2} / Q^{2}\right)$, where the impact-parameter $\mathbf{b}$ is conjugated to the transverse momentum. The threshold factor from the resummation of $\ln ^{2} u$ has been parametrized as

$$
S_{t}(u, Q)=\frac{2^{1+c\left(Q^{2}\right)} \Gamma\left(\frac{3}{2}+c\left(Q^{2}\right)\right)}{\sqrt{\pi} \Gamma\left(1+c\left(Q^{2}\right)\right)}[u(1-u)]^{c\left(Q^{2}\right)} .
$$

It was found that the nontrivial $Q^{2}$ dependence of the factor $c\left(Q^{2}\right)$ is important in the explanation of $B A B A R$ data [16]. Since the NLO QCD corrections will generate the mixed $\operatorname{logarithm} \ln u \ln \left(\zeta^{2} P^{-2} / k_{T}^{2}\right)$, both in the pion wave function and the hard kernel, the double logarithms need to be resummed. In [25], an evolution equation has been constructed to resum the mixed logarithm $\ln u \ln \left(\zeta_{P}^{2} / k_{T}^{2}\right)$. It is more convenient to perform the resummation in the moment and impact parameter space, and the result reads:

$$
\begin{gathered}
\tilde{\Phi}\left(N, b, \zeta^{2}, \mu_{f}\right)=\exp \left\{-\int_{\zeta_{0}^{2}}^{\zeta^{2}} \frac{d \tilde{\zeta}^{2}}{\tilde{\zeta}^{2}}\left[\int_{\mu_{0}(\tilde{\zeta})}^{\mu_{1}(\tilde{\zeta})} \frac{d \tilde{\mu}}{\tilde{\mu}} \lambda_{K}(\tilde{\mu}) \theta\left(\mu_{1}(\tilde{\zeta})-\mu_{0}(\tilde{\zeta})\right)\right]+\frac{3}{2} \int_{\mu_{i}}^{\mu_{f}} \frac{d \tilde{\mu}}{\tilde{\mu}} \frac{\alpha_{s}(\tilde{\mu}) C_{F}}{\pi}\right\} \tilde{\Phi}\left(N, b, \zeta_{0}^{2}, \mu_{i}\right), \\
\tilde{H}\left(N, b, \zeta^{2}, Q^{2}, \mu_{f}\right)=\exp \left\{\int_{\zeta^{2}}^{\zeta_{1}^{2}} \frac{d \tilde{\zeta}^{2}}{\tilde{\zeta}^{2}}\left[\int_{\mu_{0}(\tilde{\zeta})}^{\mu_{1}(\tilde{\zeta})} \frac{d \tilde{\mu}}{\tilde{\mu}} \lambda_{K}(\tilde{\mu}) \theta\left(\mu_{1}(\tilde{\zeta})-\mu_{0}(\tilde{\zeta})\right)\right]-\frac{3}{2} \int_{t}^{\mu_{f}} \frac{d \tilde{\mu}}{\tilde{\mu}} \frac{\alpha_{s}(\tilde{\mu}) C_{F}}{\pi}\right\} \tilde{H}\left(N, b, \zeta_{1}^{2}, Q^{2}, t\right),
\end{gathered}
$$

where $\lambda_{K}=\frac{\alpha_{s} C_{F}}{2 \pi}, \mu_{i}$ is the initial scale of the renormalization group evolution. The bounds $\zeta_{0}^{2}$ and $\zeta_{1}^{2}$ are chosen in order to eliminate the large logarithms in the initial conditions of the pion wave function and the hard kernel. The joint-resummation improved pion wave function modifies both the longitudinal and transverse momentum distributions, and both the moderate $u$ and small $b$ regions are more highlighted after resummation. In the pion TFF, by choosing appropriate $\zeta_{1}$, the hard kernel without large double logarithms reads:

$H^{(1)}\left(u, k_{T}, \zeta_{1}^{2}, Q^{2}, t\right)=-\frac{\alpha_{s}(t) C_{F}}{4 \pi}\left(3 \ln \frac{t^{2}}{u Q^{2}+k_{T}^{2}}+\ln 2+2\right)$.

When a specific model of pion wave function is employed, the joint-resummation improved wave function can be transformed to the momentum space, and the factorization formula for the pion TFF is obtained as

$$
\begin{aligned}
F^{L P}\left(Q^{2}\right)= & \frac{\sqrt{2} f_{\pi}}{3} \int_{0}^{1} d u \int_{0}^{\infty} b d b \bar{\Phi}\left(u, b, \zeta_{1}^{2}, t\right) K_{0}(\sqrt{u} Q b) \\
& \times\left[1-\frac{\alpha_{s}(t) C_{F}}{4 \pi}\left(3 \ln \frac{t^{2} b}{2 \sqrt{u} Q}+\ln 2+2\right)\right],
\end{aligned}
$$

where the expression of the wave function $\bar{\Phi}\left(u, b, \zeta_{1}^{2}, t\right)$ can be found in the Ref. [25]. In Eq. (12) all the large logarithmic terms are collected by the resummed wave function, and the hard kernel is free from large logarithms.

\section{SUBLEADING POWER CORRECTIONS}

In this section we aim at investigating the subleading power corrections to the pion TFF. As the transverse momentum of the parton of the pion meson is kept in the PQCD approach, the end-point singularity does not appear, and the factorization approach can be employed to evaluate the power suppressed contributions. As claimed in [15], a powerlike falloff of the form factor in the large $Q^{2}$ limit can be generated by both "direct" photon and 
"hadronic" photon contributions. For the direct photon contribution the hard subgraph includes both photon vertices, which starts from leading power $\left[\mathcal{O}\left(1 / Q^{2}\right)\right]$, and there exists power suppressed contributions from higher-twist pion wave functions. The contribution from hadronic photon is at most $\mathcal{O}\left(1 / Q^{4}\right)$. We will consider two kinds of subleading power corrections within the PQCD approach as follows.

\section{A. Higher-twist pion wave functions}

To evaluate the contribution from higher-twist pion wave functions, the definition of higher-twist TMD wave functions is required. For simplicity, we assume that the pion wave function can be factorized into the longitudinal and transverse parts,

$$
\Phi_{i}\left(u, k_{T}, \zeta_{0}^{2}, \mu_{i}\right)=\phi_{i}\left(u, \zeta_{0}^{2}, \mu_{i}\right) \Sigma\left(k_{T}^{2}\right) .
$$

For definiteness, the transverse momentum distribution is taken as

$$
\Sigma\left(k_{T}^{2}\right)=4 \pi \beta^{2} \exp \left(-\beta^{2} k_{T}^{2}\right)
$$

where the prefactor is introduced to obey the normalization

$$
\int \frac{d^{2} k_{T}}{(2 \pi)^{2}} \Sigma\left(k_{T}^{2}\right)=1
$$

The longitudinal momentum distribution $\phi_{i}\left(x, \zeta_{0}^{2}, \mu_{i}\right)$ is assumed to be the same as the LCDA of the pion. For the two-particle pion LCDAs, we have

$$
\begin{aligned}
\kappa_{q}\left\langle\pi(p)\left|\bar{q}(y) \gamma_{\mu} \gamma_{5} q(0)\right| 0\right\rangle & =-i f_{\pi} p_{\mu} \int_{0}^{1} d u e^{i u p \cdot y}\left[\phi(u)+y^{2} g_{1}(u)\right]+f_{\pi}\left(y_{\mu}-\frac{y^{2} p_{\mu}}{p \cdot y}\right) \int_{0}^{1} d u e^{i u p \cdot y} g_{2}(u), \\
\kappa_{q}\left\langle\pi(p)\left|\bar{q}(y) i \gamma_{5} q(0)\right| 0\right\rangle & =f_{\pi} \mu_{\pi} \int_{0}^{1} d u e^{i u p \cdot y} \phi_{p}(u), \\
\kappa_{q}\left\langle\pi(p)\left|\bar{q}(y) \sigma_{\mu \nu} \gamma_{5} q(0)\right| 0\right\rangle & =i f_{\pi} \mu_{\pi}\left(p_{\mu} y_{\nu}-p_{\nu} y_{\mu}\right) \int_{0}^{1} d u e^{i u p \cdot y} \frac{\phi_{\sigma}(u)}{6},
\end{aligned}
$$

where $\kappa_{u}=-\kappa_{d}=1 / \sqrt{2}$ for $\pi_{0}$ meson. $\phi_{\pi}(u)$ is twist-2, $\phi_{P}^{\pi}(u)$ and $\phi_{\sigma}^{\pi}(u)$ are twist-3, and $g_{1}^{\pi}(u), g_{2}^{\pi}(u)$ are twist-4.

The three-particle pion LCDAs are also defined by [26]

$$
\begin{aligned}
\kappa_{q}\left\langle\pi(p)\left|\bar{q}(y) \gamma_{\mu} \gamma_{5} g_{s} G_{\alpha \beta}(v y) q(0)\right| 0\right\rangle= & f_{\pi}\left(p_{\beta} g_{\alpha \mu}-p_{\alpha} g_{\beta \mu}-\frac{y_{\alpha} p_{\beta}-y_{\beta} p_{\alpha}}{p \cdot y} p_{\mu}\right) \\
& \times \int_{0}^{1}\left[D \alpha_{i}\right] \phi_{\perp}^{\pi}\left(\alpha_{i}\right) e^{i p \cdot y\left(\alpha_{q}+v \alpha_{g}\right)}+f_{\pi} \frac{y_{\alpha} p_{\beta}-y_{\beta} p_{\alpha}}{p \cdot y} p_{\mu} \int_{0}^{1}\left[D \alpha_{i}\right] \phi_{\|}^{\pi}\left(\alpha_{i}\right) e^{i p \cdot y\left(\alpha_{q}+v \alpha_{g}\right)} \\
\kappa_{q}\left\langle\pi(p)\left|\bar{q}(y) \gamma_{\mu} g_{s} \tilde{G}_{\alpha \beta}(v y) q(0)\right| 0\right\rangle= & i f_{\pi}\left(p_{\beta} g_{\alpha \mu}-p_{\alpha} g_{\beta \mu}-\frac{y_{\alpha} p_{\beta}-y_{\beta} p_{\alpha}}{p \cdot y} p_{\mu}\right) \\
& \times \int_{0}^{1}\left[D \alpha_{i}\right] \tilde{\phi}_{\perp}^{\pi}\left(\alpha_{i}\right) e^{i p \cdot y\left(\alpha_{q}+v \alpha_{g}\right)}+i f_{\pi} \frac{y_{\alpha} p_{\beta}-y_{\beta} p_{\alpha}}{p \cdot y} p_{\mu} \int_{0}^{1}\left[D \alpha_{i}\right] \tilde{\phi}_{\|}^{\pi}\left(\alpha_{i}\right) e^{i p \cdot y\left(\alpha_{q}+v \alpha_{g}\right)}
\end{aligned}
$$

here, we have employed the following notations for the dual field strength tensor and the integration measure:

$$
\tilde{G}_{\alpha \beta}=\frac{1}{2} \varepsilon_{\alpha \beta \rho \tau} G^{\rho \tau}, \quad \int\left[\mathcal{D} \alpha_{i}\right] \equiv \int_{0}^{1} d \alpha_{q} \int_{0}^{1} d \alpha_{\bar{q}} \int_{0}^{1} d \alpha_{g} \delta\left(1-\alpha_{q}-\alpha_{\bar{q}}-\alpha_{g}\right)
$$

We note that all three-particle LCDAs are twist-4.

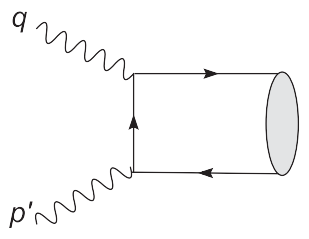

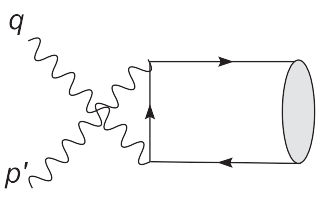

(a)

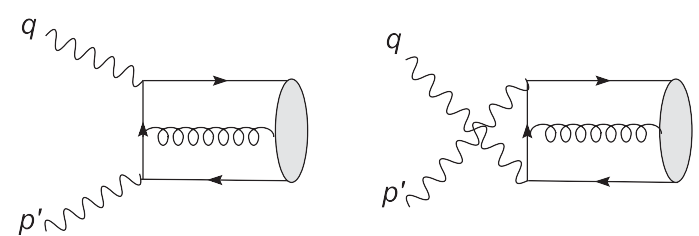

(b)

FIG. 1. Feynman diagrams of contribution of the pion transition form factor from two-particle and three-particle wave functions. 
It is straightforward to obtain the factorization formula for two-particle twist-4 contribution through evaluating the Feynman diagrams in Fig. 1(a),

$$
F^{2 P T 4}\left(Q^{2}\right)=-\frac{2 \sqrt{2} f_{\pi}}{3 Q} \int_{0}^{1} \frac{d u}{\sqrt{u}} \int_{0}^{\infty} b^{2} d b K_{1}(\sqrt{u} Q b)\left[g_{1}(u, b)+G_{2}(u, b)\right],
$$

where $G_{2}(u)=-\int_{0}^{u} g_{2}(v) d v$, and both the wave functions and the hard kernel have been transformed into the impact parameter space.

To evaluate the three-particle LCDA contribution, we need to keep the one-gluon part for the light-cone expansion of the quark propagator in the background gluon field

$$
\langle 0|T\{q(y), \bar{q}(0)\}| 0\rangle_{G} \supset i g_{s} \int \frac{d^{4} k}{(2 \pi)^{4}} e^{-i k \cdot y} \int_{0}^{1} d v\left[\frac{v y_{\mu} \gamma_{\nu}}{k^{2}}-\frac{\not k \sigma_{\mu \nu}}{2 k^{4}}\right] G^{\mu \nu}(v y),
$$

where $G^{\mu \nu}=i\left[D_{\mu}, D_{\nu}\right]$. From evaluating the diagram in Fig. 1(b), the factorization formula for three-particle twist-4 contribution reads

$$
F^{3 P T 4}\left(Q^{2}\right)=-\frac{\sqrt{2} f_{\pi}}{6 Q} \int_{0}^{1} \frac{d u}{\sqrt{u}} \int_{0}^{\infty} b^{2} d b K_{1}(\sqrt{u} Q b) \rho^{3 P T 4}\left(\alpha_{i}, b\right)
$$

where

$$
\rho^{3 P T 4}(u, b)=\int_{0}^{\bar{u}} d \alpha_{q} \int_{0}^{u} \frac{d \alpha_{\bar{q}}}{\alpha_{g}}\left[\frac{2 u-1-\alpha_{q}-\alpha_{\bar{q}}}{\alpha_{g}} \phi_{\|}^{\pi}\left(\alpha_{q}, \alpha_{\bar{q}}, 1-\alpha_{q}-\alpha_{\bar{q}}, b\right)+\tilde{\phi}_{\|}^{\pi}\left(\alpha_{q}, \alpha_{\bar{q}}, 1-\alpha_{q}-\alpha_{\bar{q}}, b\right)\right] .
$$

Employing the definition [26]

$\varphi^{T W 4}(u, b)=4\left[g_{1}(u, b)+G_{2}(u, b)\right]+\rho^{3 P T 4}(u, b)$,

we can write the overall contribution from twist-4 pion LCDAs as

$$
\begin{aligned}
& F^{T W 4}\left(Q^{2}\right) \\
& =-\frac{\sqrt{2} f_{\pi}}{6 Q} \int_{0}^{1} \frac{d u}{\sqrt{u}} \int_{0}^{\infty} b^{2} d b K_{1}(\sqrt{u} Q b) \varphi^{T W 4}(u, b)
\end{aligned}
$$

here, the Sudakov evolution factor is neglected, because there is no study on the joint-resummation effect for the higher-twist pion wave function yet. In this sense, this result is not complete in the $k_{T}$ factorization framework, but it does not matter because this contribution is actually free from endpoint singularity.

\section{B. Hadronic structure of the photon}

To investigate the contribution from the hadronic structure of the photon, the LCDAs of the photon [35] are needed. The definitions of two-particle twist- 2 and twist- 3 LCDAs are given below

$$
\begin{aligned}
\left\langle 0\left|\bar{q}(z) \sigma_{\alpha \beta} q(0)\right| \gamma(p, \lambda)\right\rangle & =i g_{\mathrm{em}} Q_{q}\langle\bar{q} q\rangle\left(p_{\beta} \epsilon_{\alpha}-p_{\alpha} \epsilon_{\beta}\right) \int_{0}^{1} d x e^{i x p \cdot z}\left[\chi(\mu) \phi_{\gamma}(x, \mu)\right], \\
\left\langle 0\left|\bar{q}(z) \gamma_{\alpha} q(0)\right| \gamma(p, \lambda)\right\rangle & =g_{\mathrm{em}} Q_{q} f_{3 \gamma} \epsilon_{\alpha} \int_{0}^{1} d x e^{i x p \cdot z} \psi_{\gamma}^{(v)}(x, \mu), \\
\left\langle 0\left|\bar{q}(z) \gamma_{\alpha} \gamma_{5} q(0)\right| \gamma(p, \lambda)\right\rangle & =\frac{1}{4} g_{\mathrm{em}} Q_{q} f_{3 \gamma} \epsilon_{\alpha \beta \rho \sigma} p^{\rho} z^{\sigma} \epsilon^{\beta} \int_{0}^{1} d x e^{i x p \cdot z} \psi_{\gamma}^{(a)}(x, \mu),
\end{aligned}
$$

where $\phi_{\gamma}(x, \mu)$ is twist-2, and $\psi_{\gamma}^{(a, v)}(x, \mu)$ is twist-3. At tree level the trace formulism is convenient to evaluate the transition matrix element, so that the following momentum space projector of photon LCDAs is useful:

$$
\begin{aligned}
M_{\alpha \beta}^{\gamma}= & \frac{1}{4} g_{\mathrm{em}} Q_{q}\left\{-\langle\bar{q} q\rangle \phi \not p \chi(\mu) \phi_{\gamma}(x, \mu)+f_{3 \gamma} \phi \psi_{\gamma}^{(v)}(x, \mu)\right. \\
& \left.-\frac{i}{8} f_{3 \gamma} \epsilon_{\mu \nu \rho \sigma}\left(\gamma^{\mu} \gamma^{5}\right) n^{\rho} \epsilon^{\nu}\left[\bar{n}^{\sigma} \frac{d}{d x} \psi_{\gamma}^{(a)}(x, \mu)-2 E_{\gamma} \psi_{\gamma}^{(a)}(x, \mu) \frac{\partial}{\partial k_{\perp \sigma}}\right]\right\}_{\alpha \beta} .
\end{aligned}
$$


Similarly, we introduce the momentum space projector of pion wave function up to two-particle twist-3

$$
M_{\delta \alpha}^{\pi}=\frac{i f_{\pi}}{4}\left\{\not p \gamma_{5} \phi_{\pi}(u)-\mu_{\pi} \gamma_{5}\left(\phi_{p}^{\pi}(u)-\frac{i}{2} \sigma_{\mu \nu} \bar{n}^{\mu} n^{\nu} \frac{\phi_{\sigma}^{\pi^{\prime}}(u)}{6}+i \sigma_{\mu \nu} p^{\mu} \frac{\phi_{\sigma}^{\pi}(u)}{6} \frac{\partial}{\partial k_{\perp \nu}}\right)\right\}_{\delta \alpha} .
$$

Since in the PQCD approach the endpoint singularity is regularized, the matrix element of pion TFF can be calculated through the convolution formula

$$
\langle\pi|\bar{q} \Gamma b| \gamma\rangle_{\mathrm{HS}}=\frac{4 \pi \alpha_{s} C_{F}}{N_{c}} \int_{0}^{1} d x \int_{0}^{\infty} b_{1} d b_{1} \int_{0}^{1} d u \int_{0}^{\infty} b_{2} d b_{2} M_{\beta \rho}^{\gamma} H_{\alpha \beta \rho \sigma}^{\Gamma} M_{\sigma \alpha}^{\pi} .
$$

The hard kernel can be obtained through calculating the Feynman diagrams in Fig. 2, and the factorization formula for the TFF reads:

$$
\begin{aligned}
F^{P H S}\left(Q^{2}\right)= & \frac{4 \pi \alpha_{s} C_{F} f_{\pi}\left(Q_{u}^{2}-Q_{d}^{2}\right)}{\sqrt{2} N_{c}} \int_{0}^{1} d x \int_{0}^{\infty} b_{1} d b_{1} \int_{0}^{1} d u \int_{0}^{\infty} b_{2} d b_{2} \\
& \times\left\{h_{e}^{a}\left(x, u, b_{1}, b_{2}\right) f_{3 \gamma} \phi_{\pi}(u) \psi_{\gamma}^{(v)}(x)+h_{e}^{b}\left(x, u, b_{1}, b_{2}\right)\left[-f_{3 \gamma} x \phi_{\pi}(u) \psi_{\gamma}^{(v)}(x)\right.\right. \\
& \left.\left.+2 \chi(\mu)\langle\bar{q} q\rangle(\mu) \mu_{\pi} \phi_{\gamma}(x) \phi_{p}^{\pi}(u)\right]\right\},
\end{aligned}
$$

with the PQCD hard function

$$
\begin{aligned}
h_{e}^{a}\left(x, u, b_{1}, b_{2}\right)= & e^{-s_{\pi}(t)-s_{\gamma}(t)}\left[\theta\left(b_{1}-b_{2}\right) I_{0}\left(\sqrt{u Q^{2}} b_{2}\right) K_{0}\left(\sqrt{u Q^{2}} b_{1}\right)\right. \\
& \left.+\theta\left(b_{2}-b_{1}\right) I_{0}\left(\sqrt{u Q^{2}} b_{1}\right) K_{0}\left(\sqrt{u Q^{2}} b_{2}\right)\right] K_{0}\left(\sqrt{x u Q^{2}} b_{1}\right) S_{t}(u), \\
h_{e}^{b}\left(x, u, b_{1}, b_{2}\right)= & e^{-s_{\pi}(t)-s_{\gamma}(t)}\left[\theta\left(b_{1}-b_{2}\right) I_{0}\left(\sqrt{x Q^{2}} b_{2}\right) K_{0}\left(\sqrt{x Q^{2}} b_{1}\right)\right. \\
& \left.+\theta\left(b_{2}-b_{1}\right) I_{0}\left(\sqrt{x Q^{2}} b_{1}\right) K_{0}\left(\sqrt{x Q^{2}} b_{2}\right)\right] K_{0}\left(\sqrt{x u Q^{2}} b_{1}\right) S_{t}(x),
\end{aligned}
$$

where the Sudakov factor $s_{\gamma}(t)$ is the same as that of a vector meson. In this part, we do not include the NLO QCD corrections, then the hard kernel does not depend on the factorization scale; thus, the form factor is dependent on the factorization scale in principle. In the PQCD approach, in order to suppress the high order corrections, the factorization scale is set to be $t=\max (\sqrt{x} Q, 1 / b)$, and we allow an error area of $t$ in the numerical evaluation. Note that the contribution of higher-twist LCDAs of the photon is also not considered in the present paper, as they are proved to be small in the previous studies [36]. The overall result for the pion TFF reads:

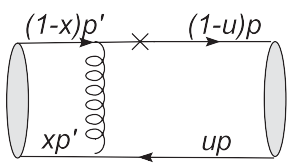

(a)

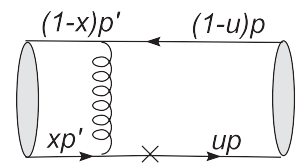

(c)

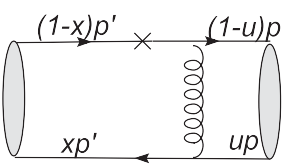

(b)

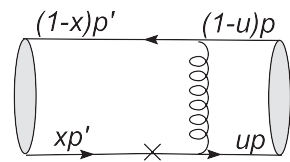

(d)
FIG. 2. Feynman diagrams of contribution of the pion transition form factor from the hadronic structure of the photon.
$F_{\gamma^{*} \gamma \rightarrow \pi^{0}}\left(Q^{2}\right)=F^{L P}\left(Q^{2}\right)+F^{T W 4}\left(Q^{2}\right)+F^{P H S}\left(Q^{2}\right)$.

\section{NUMERICAL ANALYSIS}

The leading twist pion LCDA satisfies the wellknown Efremov-Radyushkin-Brodsky-Lepage equation [1,2], which indicates that it can be expanded in terms of the Gegenbauer polynomials $C_{n}^{3 / 2}$,

$$
\phi_{\pi}(u, \mu)=6 u(1-u) \sum_{n=0}^{\infty} a_{n}(\mu) C_{n}^{3 / 2}(2 u-1),
$$

where the odd Gegenbauer moments $a_{2 n+1}$ vanish due to symmetry properties, and $a_{2 n}$ are the essential ingredients in the LCDA, with $a_{0}(\mu)=1$. Tremendous efforts have been devoted to the determinations of the lowest moment $a_{2}(\mu)$ from the calculations with the QCD sum rules and with the lattice simulations, as well as by matching the experimental data. In the numerical analysis, we will take advantage of the following models: (1) the asymptotic model, with $a_{2 n}=0(n \geq 1)$; (2) the Bakulev-MikhailovStefanis (BMS) model [40] and the platykurtic (PK) model [41], which are computed from the QCD sum rules with nonlocal condensates; (3) the Khodjamirian-MannelOffen-Wang (KMOW) model [42], which is determined by comparing the LCSR predictions for the pion 
TABLE I. The numerical values of Gegenbauer moments $a_{2}$ and $a_{4}$ in leading twist pion LCDA.

\begin{tabular}{lcccc}
\hline \hline Models & BMS & KMOW & Holographic & Platykurtic \\
\hline$a_{2}(1 \mathrm{GeV})$ & 0.20 & 0.17 & 0.15 & $0.08 \pm 0.02$ \\
$a_{4}(1 \mathrm{GeV})$ & -0.15 & 0.06 & 0.06 & -0.02 \\
\hline \hline
\end{tabular}

electromagnetic form factor with the experimental data; (4) the holographic model [43], which is motivated by the AdS/QCD correspondence. We collect the values of the Gegenbauer moments in different models in Table I. The joint-resummation improved expression for the $a_{2}$ term has been obtained in [25], and for the $a_{4}$ term, we will employ the conventional factorization formula in the $k_{T}$ factorization [see Eq. (7)].

For twist-3 pion LCDAs, we adopt [44]

$$
\begin{aligned}
& \phi_{p}^{\pi}(u)=1+0.59 C_{2}^{\frac{1}{2}}(2 u-1)+0.09 C_{4}^{\frac{1}{2}}(2 u-1) \\
& \phi_{\sigma}^{\pi}(u)=6 u(1-u)\left[1+0.11 C_{2}^{3 / 2}(2 u-1)\right] .
\end{aligned}
$$

The twist-4 pion LCDA has the following form if only the leading conformal spin contribution is kept [26]:

$$
\varphi^{T W 4}(u, \mu)=\frac{80}{3} \delta_{\pi}^{2}(\mu) u^{2}(1-u)^{2},
$$

where the normalization parameter is defined by

$$
\left\langle 0\left|g_{s} \bar{q} \tilde{G}^{\mu \nu} \gamma_{\nu} q(0)\right| \pi(p)\right\rangle=i f_{\pi} \delta_{\pi}^{2}(\mu) p^{\mu},
$$

with the renormalization-scale evolution at one loop

$$
\delta_{\pi}^{2}(\mu)=\left[\frac{\alpha_{s}(\mu)}{\alpha_{s}\left(\mu_{0}\right)}\right]^{\frac{32}{9 \beta_{0}}} \delta_{\pi}^{2}\left(\mu_{0}\right)
$$

The numerical value of $\delta_{\pi}$ will be taken as $\delta_{\pi}^{2}(1 \mathrm{GeV})=$ $(0.2 \pm 0.04) \mathrm{GeV}^{2}$ computed from the QCD sum rules [45] (see also [46]). The light-cone distribution amplitudes $\phi_{\gamma}(u), \psi^{(v, a)}(\omega, \xi)$ have been systematically studied in Ref. [35], and the expressions are quoted as follows. The two-particle twist-2 LCDA is expanded in terms of Gegenbauer polynomials,

$$
\phi_{\gamma}(x, \mu)=6 x \bar{x}\left[1+\sum_{n=2}^{\infty} b_{n}\left(\mu_{0}\right) C_{n}^{3 / 2}(2 x-1)\right],
$$

and twist-3 LCDAs in conformal expansion read:

$$
\begin{aligned}
\psi^{(v)}(\xi, \mu)= & 5\left(3 \xi^{2}-1\right) \\
& +\frac{3}{64}\left[15 \omega_{\gamma}^{V}(\mu)-5 \omega_{\gamma}^{A}(\mu)\right]\left(3-30 \xi^{2}+35 \xi^{4}\right), \\
\psi^{(a)}(\xi, \mu)= & \frac{5}{2}\left(1-\xi^{2}\right)\left(5 \xi^{2}-1\right)\left(1+\frac{9}{16} \omega_{\gamma}^{V}(\mu)-\frac{3}{16} \omega_{\gamma}^{A}(\mu)\right) .
\end{aligned}
$$

The value of the parameters used in the LCDAs of the photon are presented in Table II; among them, the scale dependent parameters are given at $\mu_{0}=1.0 \mathrm{GeV}$. These parameters should be run to the factorization scale $t$, and the evolution kernel for $\chi(\mu),\langle\bar{q} q\rangle(\mu), b_{2}(\mu), f_{3 \gamma}(\mu)$, and $\omega_{V, A}(\mu)$ have been given in [35].

Now we turn to investigate the leading power result from the joint-resummation improved PQCD approach and the contributions from various sources of subleading power corrections. In Fig. 3, the results of each kind of contribution are displayed, where the PK model is set as a default model. As the joint-resummation strongly suppressed the small- $x$ region, the moderate $x$ region $0.1<x<0.2$ gives a major contribution; thus, the pion TFF will be enhanced if the pion LCDA with two humps is adopted. The subleading power corrections start from $1 / Q^{4}$, which fall down rapidly when $Q^{2}$ increases, and are significant only at a small $Q^{2}$ region. From this figure we can see that the contributions from twist-4 pion wave functions and hadronic structure of the photon have a different sign; the cancellation between them makes the power correction investigated in this paper minor even at a small $Q^{2}$ region. This result is consistent with the investigation from light-cone sum rules [34], so that the NLP corrections in pion TFF are small (at least for the contributions considered in this paper), which is contrary to the leptonic radiative decay $B \rightarrow \gamma \ell \nu$ [37], where the NLP corrections decrease the LP result over $50 \%$. The uncertainties of the contribution from the highertwist pion wave functions and the hadronic structure of the photon are shown in Fig 4. The main source of the uncertainty are the parameters in the twist-4 pion LCDA and the LCDAs of the photon, which are presented in Table II, as well as the factorization scale $t$, which is allowed a float up or down 20\%. The contribution from the twist-4 pion LCDA was also investigated in Refs. [32,33], where the uncertainty caused by the variation of $\delta_{\pi}^{2}$ is about $3 \%$, and this result is consistent with ours. Our current

TABLE II. Numerical value of the parameters in the LCDAs of the photon.

\begin{tabular}{lccr}
\hline \hline Parameter & $\chi(1 \mathrm{GeV})$ & $\langle\bar{q} q\rangle(1 \mathrm{GeV})$ & $b_{2}(1 \mathrm{GeV})$ \\
\hline Value & $(3.15 \pm 0.03) \mathrm{GeV}^{-2}$ & $-\left[\left(256_{-16}^{+14}\right) \mathrm{MeV}\right]^{3}$ & $0.07 \pm 0.07$ \\
Parameter & $f_{3 \gamma}(1 \mathrm{GeV})$ & $\omega_{\gamma}^{V}(1 \mathrm{GeV})$ & $\omega_{\gamma}^{A}(1 \mathrm{GeV})$ \\
Value & $-(4 \pm 2) \times 10^{-3} \mathrm{GeV}^{2}$ & $3.8 \pm 1.8$ & $-2.1 \pm 1.0$ \\
\hline \hline
\end{tabular}




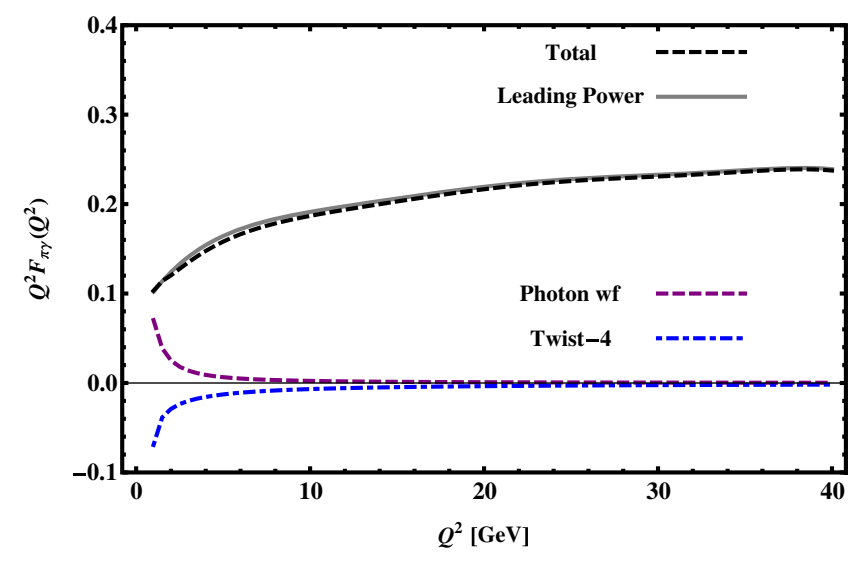

FIG. 3. Contributions of leading power and subleading power corrections to the pion TFF.

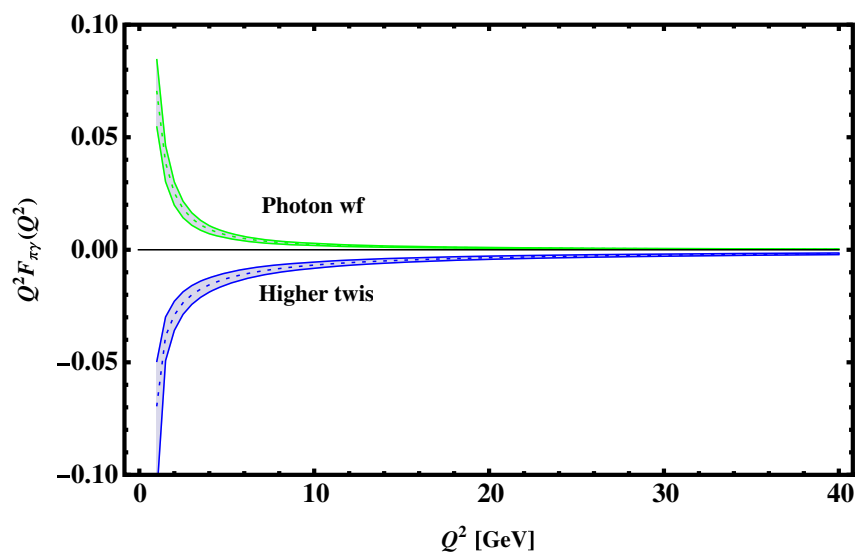

FIG. 4. Uncertainties of the contribution from subleading power corrections to the pion TFF.

result indicates that the power corrections can hardly modify the leading power prediction from the joint-resummation improved PQCD approach; thus, the conclusion in Ref. [25] still holds.

To investigate how the dependence of the pion TFF on the Gegenbauer moments appears in the leading twist pion LCDA, the results of $Q^{2} F_{\gamma^{*} \gamma \rightarrow \pi^{0}}\left(Q^{2}\right)$ from the asymptotic model, the BMS model, the PK model, the KMOW model, and the holographic model are plotted in Fig. 5. For the asymptotic model, our result is approaching the limit $\sqrt{2} f_{\pi}$ as expected, while for the models with large Gegenbauer moment $a_{2}$, the results of the pion TFF even overshoot the $B A B A R$ data as the joint-resummation enhances the moderate- $x$ region. In the BMS model, the $a_{4}$ term has the opposite sign with the $a_{2}$ term, but its contribution is small in our formalism, which is different from the LCSR result. The result from the models with a smaller $a_{2}$ (such as the PK model) is more comparable with $B A B A R$ and Belle data. This tendency indicates that the model with a smaller $a_{2}$ will favor the Belle data well (As we do not perform

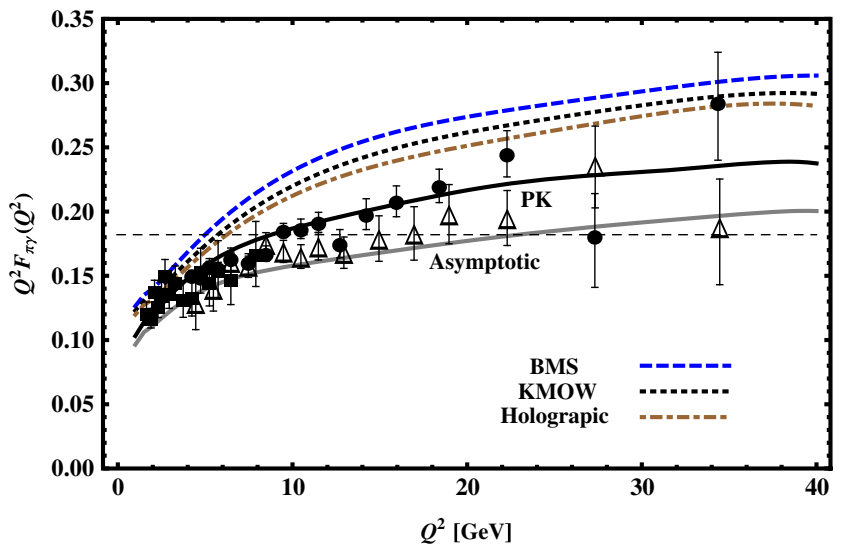

FIG. 5. Comparison of different models of leading twist pion LCDA; the experimental data are from CLEO (squares), BABAR (dots), and Belle (triangles).

joint-resummation with respect to the $a_{4}$ term, the current result does not mean that the model such as BMS should be ruled out.). Since the pion TFF is sensitive to the Gegenbauer moment $a_{2}$, it provides a good platform to extract the nonperturbative information on the shape of the leading twist pion LCDA.

The comparison between our prediction with uncertainty and the experimental data is presented in Fig. 6, where the CLEO [47], BABAR [8] and Belle [9] data are shown. In this figure we also display the fitted function by Belle and $B A B A R$ (here we only quote the central value),

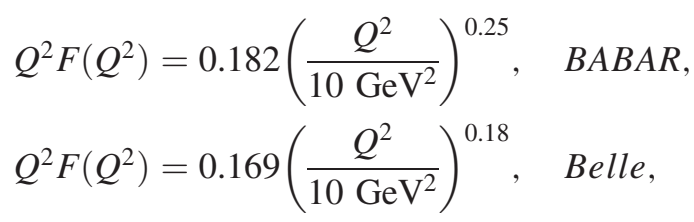

which are plotted with the thick line $(B A B A R)$ and the dashed line (Belle). For the twist-2 pion LCDA we employ

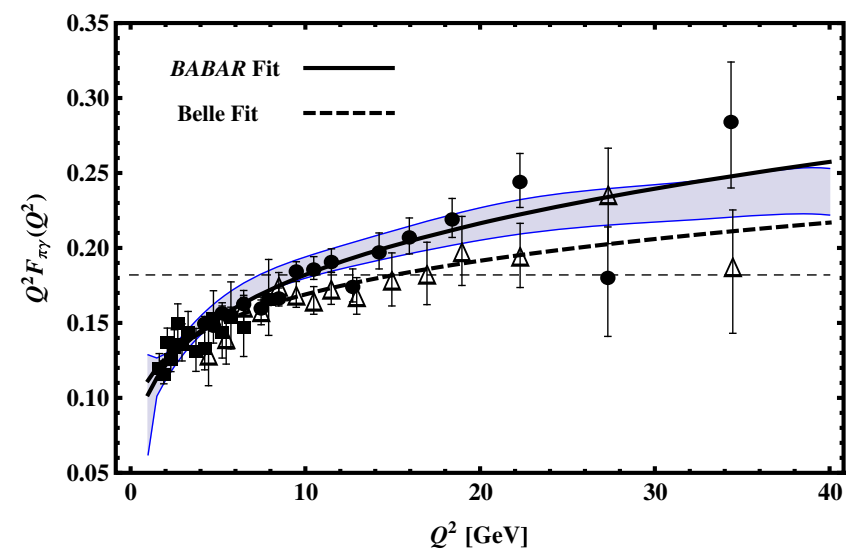

FIG. 6. Comparison between theoretical prediction and experimental data; the experimental data are from CLEO (squares), $B A B A R$ (dots), and Belle (triangles). 
the PK model with the parameters given in Table I. The uncertainties are mainly from $a_{2}$ at a large $Q^{2}$ region, and from $\delta_{\pi}$ and the parameters in photon LCDA at a small $Q^{2}$ region. It can be seen from Fig. 6 that the prediction from the PK model is consistent with $B A B A R$ data at intermediate $Q^{2}$, and is between $B A B A R$ and Belle fitted curves when $Q^{2}$ is getting large. Because of the cancellation between the contribution from twist-4 pion LCDA and photon LCDA, the subleading power contribution only changes the leading power result slightly, even at a small $Q^{2}$ region, and the prediction with the $\mathrm{PK}$ model is consistent with the CLEO and BABAR data. We emphasize that there is a large theoretical uncertainty when $Q^{2}<2 \mathrm{GeV}^{2}$, and the perturbation calculation is not reliable at this region; thus, one needs not to take it seriously. Although in the present work the NLP contribution is negligible, it is too early to draw the conclusion that the power suppression contribution is not important in this process, since this work is not a systematical study on the power corrections within the framework of effective theory.

\section{SUMMARY}

In this paper, within the framework of the PQCD approach based on $k_{T}$ factorization, we studied the high power corrections to the pion TFF. For the higher power contributions, we considered the effects from higher-twist pion wave functions, including two-particle and threeparticle twist- 4 wave functions of the pion, and the hadronic structure of the photon. In the PQCD approach, the transverse momentum of partons inside the pion and "hadronic" photon regularized end-point singularity existing in collinear factorization. The numerical result indicates that there exists a strong cancellation effect between the two kinds of NLP contributions; thus, the NLP corrections do not change the leading power result manifestly. We compared the result from different models of leading twist pion LCDA and found that the large $a_{2}$ will even overshoot the anomalous $B A B A R$ data; thus, a smaller $a_{2}$ can accommodate the anomalous data from $B A B A R$, or agree with results from Belle according to the specific choice. So far there is no confirmed conclusion on the two experimental results; we hope the Belle-II experiments can test our result and give the final conclusion in future. Furthermore, we also note that the pion TFF sharply depends on the leading twist pion LCDA; it provides a good platform to extract the information of the pion wave function, which can also be compared with the future lattice simulation with the help of quasi parton distribution amplitude $[48,49]$. The power suppressed contributions considered in this paper are only from some specific sources; to perform a systematical study on this process we need to analyze the complete NLP operator [50], based on soft-collinear effective theory [51,52]. This work is left for a future study.

\section{ACKNOWLEDGMENTS}

This work was supported in part by the National Natural Science Foundation of China under the Grants No. 11705159, No. 11447032, and No. 11575151; and the Natural Science Foundation of Shandong province under the Grant No. ZR2018JL001 and No. ZR2016JL001.
[1] G. P. Lepage and S. J. Brodsky, Phys. Rev. D 22, 2157 (1980).

[2] A. V. Efremov and A. V. Radyushkin, Phys. Lett. 94B, 245 (1980).

[3] A. Duncan and A.H. Müeller, Phys. Lett. 90B, 159 (1980).

[4] F. del Aguila and M. K. Chase, Nucl. Phys. B193, 517 (1981).

[5] E. Braaten, Phys. Rev. D 28, 524 (1983).

[6] E. P. Kadantseva, S. V. Mikhailov, and A. V. Radyushkin, Yad. Fiz. 44, 507 (1986) [Sov. J. Nucl. Phys. 44, 326 (1986)].

[7] B. Melic, D. Müeller, and K. Passek-Kumericki, Phys. Rev. D 68, 014013 (2003).

[8] B. Aubert et al. (BABAR Collaboration), Phys. Rev. D 80, 052002 (2009).

[9] S. Uehara et al. (Belle Collaboration), Phys. Rev. D 86, 092007 (2012).

[10] P. Masjuan, Phys. Rev. D 86, 094021 (2012).
[11] M. Hoferichter, B. Kubis, S. Leupold, F. Niecknig, and S. P. Schneider, Eur. Phys. J. C 74, 3180 (2014).

[12] A. Gérardin, H. B. Meyer, and A. Nyffeler, Phys. Rev. D 94, 074507 (2016).

[13] A. V. Radyushkin, Phys. Rev. D 80, 094009 (2009).

[14] M. V. Polyakov, JETP Lett. 90, 228 (2009).

[15] S. S. Agaev, V. M. Braun, N. Offen, and F. A. Porkert, Phys. Rev. D 83, 054020 (2011).

[16] H.-n. Li and S. Mishima, Phys. Rev. D 80, 074024 (2009).

[17] S. Nandi and H. n. Li, Phys. Rev. D 76, 034008 (2007).

[18] I. V. Musatov and A. V. Radyushkin, Phys. Rev. D 56, 2713 (1997).

[19] X. G. Wu and T. Huang, Phys. Rev. D 82, 034024 (2010).

[20] J. C. Collins, Acta Phys. Pol. B 34, 3103 (2003).

[21] I. O. Cherednikov and N. G. Stefanis, Nucl. Phys. B802, 146 (2008).

[22] A. Bacchetta, D. Boer, M. Diehl, and P. J. Mulders, J. High Energy Phys. 08 (2008) 023. 
[23] J. Collins, Camb. Monogr. Part. Phys. Nucl. Phys. Cosmol. 32, 1 (2011).

[24] H. n. Li and Y. M. Wang, J. High Energy Phys. 06 (2015) 013.

[25] H. N. Li, Y. L. Shen, and Y. M. Wang, J. High Energy Phys. 01 (2014) 004.

[26] A. Khodjamirian, Eur. Phys. J. C 6, 477 (1999).

[27] S. S. Agaev, V. M. Braun, N. Offen, and F. A. Porkert, Phys. Rev. D 86, 077504 (2012).

[28] A. P. Bakulev, S. V. Mikhailov, and N. G. Stefanis, Phys. Rev. D 67, 074012 (2003).

[29] N. G. Stefanis, A. P. Bakulev, S. V. Mikhailov, and A. V. Pimikov, Phys. Rev. D 87, 094025 (2013).

[30] A. P. Bakulev, S. V. Mikhailov, A. V. Pimikov, and N. G. Stefanis, Phys. Rev. D 84, 034014 (2011).

[31] A. P. Bakulev, S. V. Mikhailov, A. V. Pimikov, and N. G. Stefanis, Phys. Rev. D 86, 031501 (2012).

[32] S. V. Mikhailov, A. V. Pimikov, and N. G. Stefanis, Phys. Rev. D 93, 114018 (2016).

[33] C. Ayala, S. V. Mikhailov, and N. G. Stefanis, Phys. Rev. D 98, 096017 (2018).

[34] Y. M. Wang and Y. L. Shen, J. High Energy Phys. 12 (2017) 037.

[35] P. Ball, V. M. Braun, and N. Kivel, Nucl. Phys. B649, 263 (2003).

[36] Y. M. Wang and Y. L. Shen, J. High Energy Phys. 05 (2018) 184.

[37] Y. L. Shen, Z. T. Zou, and Y. B. Wei, Phys. Rev. D 99, 016004 (2019).
[38] Y. L. Shen, J. Gao, C. D. Lü, and Y. Miao, Phys. Rev. D 99, 096013 (2019).

[39] M. Nagashima and H.-n. Li, Phys. Rev. D 67, 034001 (2003).

[40] A. P. Bakulev, S. V. Mikhailov, and N. G. Stefanis, Phys. Lett. B 508, 279 (2001); 590, 309(E) (2004).

[41] N. G. Stefanis, Phys. Lett. B 738, 483 (2014).

[42] A. Khodjamirian, T. Mannel, N. Offen, and Y.-M. Wang, Phys. Rev. D 83, 094031 (2011).

[43] S. J. Brodsky and G. F. de Teramond, Phys. Rev. D 77, 056007 (2008).

[44] H. n. Li, Y. L. Shen, and Y. M. Wang, Phys. Rev. D 85, 074004 (2012).

[45] V. A. Novikov, M. A. Shifman, A. I. Vainshtein, M. B. Voloshin, and V. I. Zakharov, Nucl. Phys. B237, 525 (1984).

[46] P. Ball, V. M. Braun, and A. Lenz, J. High Energy Phys. 05 (2006) 004.

[47] J. Gronberg et al. (CLEO Collaboration), Phys. Rev. D 57, 33 (1998).

[48] W. Wang, S. Zhao, and R. Zhu, Eur. Phys. J. C 78, 147 (2018).

[49] Y. S. Liu, W. Wang, J. Xu, Q. A. Zhang, S. Zhao, and Y. Zhao, Phys. Rev. D 99, 094036 (2019).

[50] I. Z. Rothstein, Phys. Rev. D 70, 054024 (2004).

[51] C. W. Bauer, S. Fleming, D. Pirjol, and I. W. Stewart, Phys. Rev. D 63, 114020 (2001).

[52] M. Beneke, A. P. Chapovsky, M. Diehl, and T. Feldmann, Nucl. Phys. B643, 431 (2002). 